\title{
AKR1B7 (mouse vas deferens protein) is dispensable for mouse development and reproductive success
}

\author{
C Baumann, B Davies, M Peters ${ }^{1}$, U Kaufmann-Reiche ${ }^{1}, \mathrm{M}_{\text {Lessl }}{ }^{1}$ and $\mathrm{F}$ Theuring \\ Center for Cardiovascular Research/Institute of Pharmacology and Toxicology, Charité University Medicine Berlin, \\ Hessische Strasse 3-4, 10115 Berlin, Germany and ${ }^{1}$ Research Laboratories of Bayer Schering Pharma AG, \\ Muellerstrasse 178, 13342 Berlin, Germany
}

Correspondence should be addressed to F Theuring; Email: franz.theuring@charite.de

C Baumann is now at Department of Clinical Studies, School of Veterinary Medicine, Center for Animal Transgenesis and Germ Cell Research, University of Pennsylvania, Kennett Square, Pennsylvania, USA

B Davies is now at Wellcome Trust Centre for Human Genetics, Roosevelt Drive, Oxford OX3 7BN, UK

\begin{abstract}
AKR1B7 (aldo-keto reductase family 1, member 7; also known as mouse vas deferens protein) is a member of the AKR superfamily, and has been suggested to play a role in detoxifying processes on account of its preferred substrates, 4-hydroxynonenal and isocaproaldehyde. High levels of protein expression were found in the vas deferens and the adrenal gland, where sustained expression is dependent on androgen or ACTH respectively. Recently, a remarkable induction of AKR1B7 expression has been reported in the ovary following exogenous injections of LH. In the present study, we confirm this regulation physiologically during the estrous cycle, observing $A k r 1 b 7$ expression to be restricted to the theca and stromal cells of the proestrus ovary. To further investigate the role of this detoxifying enzyme in both male and female reproduction, we generated knockout mice deficient in AKR1B7. Although AKR1B7 expression in the vas deferens is considerable and tightly regulated in the ovary of wild-type animals, homozygous mutant animals were found to be viable and no reproductive phenotype was observed. Ovarian follicle maturation and spermatozoa parameters remained normal in the absence of this protein. The determination of serum progesterone revealed an increase in hormone concentration in metestrus, while progesterone was found to be decreased in the estrus phase of the cycle in knockout females. Reproduction (2007) 134 97-109
\end{abstract}

\section{Introduction}

Aldo-keto reductases (AKRs) are NADPH-dependent oxidoreductases, which convert a broad variety of carbonyl compounds into their corresponding alcohols (Bohren et al. 1989). Monosaccharides, steroids, prostaglandins, xenobiotics as well as aliphatic and aromatic aldehydes belong to the preferred substrates (Jez et al. 1997). The AKRs comprise a diverse gene family consisting of at least 119 members to date. A multitude of species ranging from higher vertebrates and plants to protozoa, eubacteria, fungi, and archaebacteria has been found to express AKRs. In accordance with the broad substrate specificity reported for members of this superfamily, the individual enzymes show divergent functional specialization (Hyndman et al. 2003).

The initial discovery of AKR1B7 (AKR family 1, member 7; also known as mouse vas deferens protein) as the major protein of the murine vas deferens (Taragnat et al. 1986) has been causative for its designation.
Subsequently, a high level of expression was reported in the zona fasciculata of the adrenal cortex (Lau et al. 1995, Aigueperse et al. 1999) in ovaries following stimulation with a luteinizing hormone (LH) analog (Brockstedt et al. 2000) and, to a lesser extent, in several other organs, e.g., kidney, intestine, eye, and seminal vesicle (Lau et al. 1995). Extensive investigation of the gene's transcriptional regulation found the vas deferens expression to be androgen-dependent (Taragnat et al. 1988, Martinez et al. 2001) and similarly, adrenal expression was found to be dependent on corticotrophin (adrenocorticotrophin (ACTH); Lefrancois-Martinez et al. 1999, Martinez et al. 2001). We previously demonstrated a remarkable 300-fold induction of AKR1B7 protein in ovarian theca cells following application of human chorionic gonadotropin (hCG). AKR1B7 protein is only detectable within a timeframe of 1.5-14 h after hCG treatment, thus suggesting an important role for this AKR in ovulatory processes (Brockstedt et al. 2000). The promoter region of 
Akr1b7 has been shown to contain two sequence motives with high homology to the cAMP response element (Aigueperse et al. 1999). The rapid induction of Akr1b7 mRNA within $1.5 \mathrm{~h}$ post-hCG might, therefore, be a direct effect of CAMP on the promoter (ACTH as well as $\mathrm{LH}$ exert their actions mainly via cAMP). Moreover, expression of $A k r 1 b 7$ in the adrenal gland is dependent on transcription factors like NR5A1 (also known as SF1) and CCAAT/enhancer binding protein (C/EBP)- $\beta$ (Aigueperse et al. 1999), which are additionally involved in the regulation of expression of steroidogenic genes.

A regulation by the $\mathrm{LH} / \mathrm{hCG}$ surge has also been shown for cytochrome P450scc (cholesterol side-chain cleavage enzyme, Cyp11a1) mRNA (fivefold increase within $7 \mathrm{~h}$ ) in theca and granulosa cells (Goldring et al. 1987, Richards et al. 1987). Taken together, Akr1b7 expression coincides with the induction of cytochrome P450scc (Cyp11a1) mRNA expression in theca cells and in the adrenal gland and is regulated by inducers of steroidogenic activity.

In vitro analysis of substrate specificity revealed a preference of AKR1B7 protein for isocaproaldehyde as well as for 4-hydroxynonenal (4-HNE; Lefrancois-Martinez et al. 1999), two highly toxic and harmful compounds (Dianzani et al. 1999, Pizzimenti et al. 2002) whose detoxification into less reactive metabolites is of considerable importance for cellular survival. Isocaproaldehyde accumulates as a byproduct in one of the first steps of steroidogenesis during cholesterol side-chain cleavage (Constantopoulos et al. 1966, Burstein \& Gut 1971, Esterbauer et al. 1991). In concordance with its substrate specificity, the adrenal and ovarian sites of AKR1B7 expression are important sources of steroidogenic hormones such as glucocorticoids, progestins and androgens, and thus a role in detoxifying isocaproaldehyde in these tissues is hypothesized. Moreover, Akr1b7 expression could not be detected in granulosa cells, the corpus luteum, or the placenta. The vas deferens in contrast is not known to be steroidogenic; however, here $A k r 1 b 7$ expression is exceptionally high. Whether the protein fulfills several distinct functions depending on the expressing cell type or organ is not known. A general function as a detoxifying protein in steroidogenesis is indeed unlikely; however, several lines of evidence suggest such a role in theca cells of the ovary and in the adrenal gland.

The formation of the highly reactive 4-HNE occurs through reactive oxygen species-induced peroxidation of polyunsaturated fatty acids (Yi et al. 1997, Comporti 1998). Since the membranes of spermatozoa, transported by the vas deferens, contain higher concentrations of polyunsaturated fatty acids in comparison with other cell types (Aitken 1994), the remarkable strong expression of AKR1B7 within this organ may represent a protective mechanism against this toxic aldehyde, ensuring sperm viability.
The identification of AKR1B7 as a potent enzyme catalyzing 4-HNE as well as isocaproaldehyde in vitro together with its spatial and temporal expression pattern within the ovary, vas deferens, and furthermore within the adrenal gland suggests a potential physiological role for this enzyme in reproductive function. Using the Cre/loxP technology, we prepared knockout animals $\left(A k r 1 b 7^{\text {tm1.1Fth }} / A k r 1 b 7^{\text {tm1.1Fth }}\right)$ and ascertained the effect of targeted deletion of the $A k r 1 b 7$ gene on reproductive physiology.

\section{Materials and Methods}

\section{Animals}

Mice used throughout this study were kept under controlled temperature $\left(20-22{ }^{\circ} \mathrm{C}\right)$, with a $12 \mathrm{~h}$ light:12 h darkness cycle, relative humidity of $50-70 \%$, and food and water ad libitum. All animal studies were performed in compliance with the Animal Care and Use Committee guidelines of the Land Berlin, Germany.

\section{In situ hybridization}

Vaginal cytology was used for the determination of the estrus cycle according to the method described by Butcher et al. (1974). LH concentrations in proestrus reach maxima ( $\mathrm{LH}$ surge) at $\sim 1800 \mathrm{~h}$ (Bailey 1987). Therefore, vaginal smears were obtained daily at $1800 \mathrm{~h}$ over a period of 20 days and, immediately after that tissues were collected from animals at diestrus, proestrus, estrus, and metestrus. At proestrus stage, tissue preparation was additionally conducted 6 and $14 \mathrm{~h}$ postassumed LH surge. mRNA expression was exclusively assessed on ovaries from animals showing at least three consecutive 4-day estrous cycles.

Localization of $A k r 1 b 7$ mRNA in tissue sections was performed by non-radioactive in situ hybridization. Tissue fixation, template preparation, in situ hybridization, and immunological detection were conducted as described earlier (Brockstedt et al. 2000).

\section{Gene targeting in embryonic stem (ES) cells}

Mouse genomic PAC clones containing the murine $A k r 1 b 7$ gene were obtained from a 129 Ola mouse genomic library (German Resource Center $\mathrm{GmbH}$, Berlin, Germany) using a mouse cDNA probe corresponding to the exons 2-4 of the $A k r 1 b 7$ gene. A $3 \mathrm{~kb}$ Dral genomic fragment corresponding to exons 2-4 was cloned into pBluescript KS (Stratagene) 3' of a previously inserted loxP sequence, creating the plasmid pAKR1B7 loxP. The $5^{\prime}$ homology arm, a $2.4 \mathrm{~kb}$ genomic fragment corresponding to a region spanning intron 1, was amplified by PCR (Expand Long Template PCR System, Boehringer Mannheim) using the primers 5'-gAAgCTTAggCCAgCATTATCACCTgTTgTCag-3' and 5'-TAAgCTTgCAATgTgATTTgAACTCTGCTATgg - $3^{\prime}$ 
(containing restriction enzyme-cleavage sites for HindIII) and was cloned into the pAKR1B7loxP plasmid, $5^{\prime}$ of the loxP site via HindIII, creating the plasmid pAKR1B7loxP5'. The $3 \mathrm{~kb} \mathrm{3'}$ homology arm, corresponding to a region containing exons 5-8, was amplified using the primers 5'-TgTCgACAAACTCTCCTCCCAATgACTCTCC-3' and 5'-ggTgTAggTGTgCTAAgTATgTATggCCTg-3' (containing restriction enzyme-cleavage sites for Sall and Xhol respectively) and was cloned into a Xhol site of the ploxPneo vector, a plasmid containing a floxed selection cassette, comprising the neomycin phosphotransferase gene under independent promoter control $(P g k 1)$ and an SV40 polyadenylation sequence (Yang et al. 1998). This floxed cassette together with the $3^{\prime}$ targeting arm was then inserted $3^{\prime}$ of the Dral fragment creating the plasmid pAKR1B7-KO. The targeting vector was completed by the insertion of two copies of a herpes simplex virus thymidine kinase ( $T$ k 1 ) gene, serving as negative selection cassette (Smith 1991; Fig. 2A). The prepared vector was linearized by Xhol and transfected into E14.1 ES cells. The resulting neomycin-/gancyclovirresistant colonies were screened for homologous recombination within the $5^{\prime}$ homologous region by PCR. A $2.3 \mathrm{~kb}$ amplification product was obtained from homologous recombined clones using the primers 5'-CagAgCATCTATCTgCCTTgggTTTgA-3', a forward primer hybridizing to a sequence in exon 1 and $5^{\prime}$-gggTTATTgAATATgATCggAATTCgA- $3^{\prime}$, a reverse primer hybridizing to the loxP site $5^{\prime}$ of exons 2-4. Homologous recombination within the $5^{\prime}$ and $3^{\prime}$ homology arms was confirmed using Southern analysis (data not shown). Three independent clones showing correct homologous recombination were expanded and transiently transfected with a Cre recombinase expression vector (Taniguchi \& Hayashi 1998) in order to remove the selection marker together with the genomic region corresponding to exons 2-4. Analysis of G418sensitive clones yielded a variety of recombined clones suitable for the generation of a constitutive knockout (Type III) as well as for the generation of a floxed allele (Type I; deletion of the selection cassette alone). Screening of the resulting clones was performed by Southern analysis. A PCR generated probe, corresponding to the $5^{\prime}$ region of intron 1 , specifically detected fragments of $11 \mathrm{~kb}$ (wild type) and $9 \mathrm{~kb}$ (Type III recombined) within Bglll digested genomic DNA.

\section{Generation of knockout mice}

Type III recombined ES cell clones were injected into the blastocysts of $\mathrm{C} 57 \mathrm{BL} / 6$ mice and the resulting chimeric males were mated to C57BL/6 females. Genotyping of the progeny was performed by PCR analysis of tail-tip DNA using a forward primer hybridizing to intron 1 ( $5^{\prime}$-TgC CTC AgC CTC TCA CA-3') and two reverse primers, one hybridizing to the floxed region, directly upstream of exon 2 which is deleted in the knockout allele (Type III recombined; $5^{\prime}$ gCA ggg CCC ATA ACT Tg $3^{\prime}$ ) and the other hybridizing downstream of exon 4 ( $5^{\prime}$ TTg ggA gAA gAA AAC ATA CAC T $3^{\prime}$ ). The progeny of heterozygous matings was subjected to confirmatory Southern analysis according to the strategy described earlier. The resulting knockout allele has the designation $A k r 1 b 7^{\text {tm1.1Fth }}$ (MGI: 3629297).

\section{Northern and western analysis}

Total RNA, isolated from freshly prepared vasa deferentia, adrenal glands, and ovaries ( $8 \mathrm{~h}$ post-hCG stimulation) using Trizol (Invitrogen Life Technologies), was electrophoretically separated on a $1 \%$ agarose gel in the presence of $18 \%$ formaldehyde and transferred to Hybond $\mathrm{N}$ membranes (Amersham). Hybridization according to standard conditions was performed with a ${ }^{32} \mathrm{P}$-radiolabeled probe corresponding to nucleotides 843-1183 (exons 9 and 10) of the submitted $A k r 1 b 7$ sequence (Accession number: J05663). Immature mice at 3 weeks of age were injected intraperitoneally with a single dose of $20 \mathrm{IU}$ follicle-stimulating hormone (Intergonan, Intervet, Unterschleissheim, Germany) and $48 \mathrm{~h}$ later, with $10 \mathrm{IU}$ hCG (Ovogest, Intervet) to robustly induce $A k r 1 b 7$ expression.

Expression analysis of $A k r 1 b 8$ by northern blotting in adrenal glands and vasa deferentia was conducted using a Akr1b8-specific cDNA probe, spanning a region in the $3^{\prime}$ UTR between 1049 and $1272 \mathrm{bp}$ of the submitted Akr1b8 sequence (Accession number: U04204).

For western analysis, a synthetic peptide antigen comprising the amino acid sequence of murine AKR1B7 between residues 121 and 141 (ALLPKDNKGKVLLSKSTFLDA) was coupled to keyhole limpet hemocyanian via an additional cysteine at the carboxy terminus (Schnolzer et al. 1992) and was used to produce a polyclonal antiserum in rabbits. Tissue samples from vas deferens, adrenal glands, and ovaries $8 \mathrm{~h}$ following an ovulatory hCG injection were pulverized in liquid nitrogen and resuspended in $250 \mathrm{mM}$ Tris$\mathrm{HCl}(\mathrm{pH} 7.4), 0.4 \mathrm{mM}$ phenylmethylsulfonyl fluoride. Soluble tissue extracts $(20 \mu \mathrm{g})$ were subjected to SDSPAGE on $12 \%$ acrylamide gels for electrophoretic separation, according to Laemmli (1970) and subsequently transferred to PVDF membranes (Bio-Rad). The membranes were incubated for $1 \mathrm{~h}$ at room temperature in Tris-buffered saline solution containing $5 \%$ skimmed milk and incubated over night at $4{ }^{\circ} \mathrm{C}$ with AKR1B7-specific antipeptide antiserum (1:1000) recognizing an epitope within the deletion domain. Peroxidase-conjugated anti-rabbit IgG secondary antibodies were added at a 1:2500 for $1 \mathrm{~h}$ at room temperature. Peroxidase activity was detected with the ECL System (ECL, Amersham Pharmacia Biotech).

\section{Histology, organ weight determination, and assessment of follicle maturation stages}

Organ weight of the ovaries, testes, epididymides, and adrenal glands was determined immediately after dissection from the body cavity and removal of connective tissue. 
Animals used were 20 weeks of age. Tissues were then fixed for $24 \mathrm{~h}$ in $4 \%$ PBS-buffered paraformaldehyde for histological analysis. After dehydration, paraffin embedding and sectioning, hematoxylin and eosin staining were performed according to standard procedures. In order to evaluate the distribution pattern of different folliclematuration stages, $5 \mu \mathrm{m}$ serial sections of the entire organ of knockout animals $(n=4)$ and wild-type littermates $(n=4)$ at metestrus phase of the cycle were prepared. Evaluation of follicle maturation stages in serial sections was restricted to every fourth section using the nucleus of the oocyte as a guide. Under the assumption of a $20 \mu \mathrm{m}$ diameter of the nucleus, this procedure was chosen to avoid double evaluation of single follicles. This principle was also adhered to for the evaluation of atretic follicles. Only follicles containing an intact oocyte with a nucleus were considered, and follicles with fragmented oocytes and disrupted nuclei were eliminated. The presence of pyknotic granulosa cell nuclei (shriveled cells with fragmented dark nuclei and apoptotic bodies) and/or the degeneration of the oocyte nucleus served as criteria of atresia. Since morphological signs of atresia were not observed in follicles from stage 3 to 4, the data presented refer exclusively to follicles of stages 5-8. Follicle maturation stages were classified according to Pedersen \& Peters (1968) and Hegele-Hartung et al. (2004). Type 3a and $3 \mathrm{~b}$ follicles are characterized by a single cell layer of granulosa cells surrounding the oocyte, whereas type 4 follicles comprise oocytes with two layers of granulosa cells. Type $5 \mathrm{a}$ and $5 \mathrm{~b}$ follicle show several layer of granulosa cells around the oocyte and are classified as preantral follicles. The beginning of the antrum formation marks type 6 follicles, followed by further advanced stages of antral follicles (type 7 and type 8).

\section{Cumulative estrous cycle staging}

For the analysis of estrous cycle stage durations, ovaries were collected from 6 - to 10 -week-old knockout $(n=12)$ and wild-type $(n=12)$ females immediately after assessment of the estrous cycle stage (daily between 1700 and 1800 h). Classification was performed according to Rugh (1968). Accordingly, proestrus vaginal smears contain mainly epithelial cells that are round and visibly nucleated. Cells from the estrus phase are cornified squamous epithelial cells with a highly granular cytoplasm and an irregular shape that retain a visible nucleus in early estrus; however, they lack a visible nucleus in the later estrus stage of the cycle. Small leukocytes with granular cytoplasm predominate in the metestrus and diestrus phases.

\section{Fertility studies}

To compare fertility parameters of knockout females ( $n=5-10 /$ age group) versus wild-type littermate controls ( $n=5-6 /$ age group), animals at 30 days of age (age group 1) or at 5 months of age (age group 2) were mated with 10-week-old male C57BL/6 animals. The second age group was chosen to increase the number of 4- to 5-day estrous cycles experienced before the first pregnancy, to assess whether continuous pregnancy beginning immediately after puberty might mask an effect of the gene deletion. Knockout males $(n=10)$ and wild-type littermate controls $(n=13)$ which are 6-weekold were mated with the 8-week-old NMRI (outbred Swiss mouse, Naval Medical Research Institute, USA) females. All matings were performed at a ratio of 1:1. Breeding pairs were maintained together for a period of 10-15 months and successful mating was determined by the observation of a vaginal plug. Breeding efficiency was determined by analysis of average litter sizes and frequencies of parturition.

\section{Analysis of spermatozoa}

Spermatozoa were collected unilaterally from the cauda epididymis and the vas deferens respectively of knockout $(n=8)$ and wild-type littermate control $(n=10)$ animals at the age of 22 weeks. The cauda region of the epididymis and the entire vas deferens were cut open and incubated for $10 \mathrm{~min}$ in prewarmed PBS at $37^{\circ} \mathrm{C}$ to allow the spermatozoa to emerge as described previously (Davies et al. 2004). A homogenous suspension was then diluted, spread over a microscope slide, and spermatozoa were counted and assessed. The motility was defined as the percentage of motile spermatozoa present in a defined volume. Mobility as a qualitative measure was visually assessed, using an arbitrary scale according to recommendations by the World Health Organization (1987); $(0-3 ; 0=$ no overall motility; $3=$ high overall motility). The analysis of morphological characteristics included a quantification of headless, angulated, and head-to-head agglutinated spermatozoa (in percent).

\section{Analysis of serum steroid hormone concentrations}

Serum hormone concentrations from animals at metestrus and estrus stage of the cycle (assessed daily between 1700 and $1800 \mathrm{~h}$ ) were determined using the DSLACTIVE Progesterone Coated Tube Kit (DSL-5000, Diagnostic Systems Laboratories Inc., Webster, Texas, USA) and the DSL-4400 Estradiol RIA Kit (Diagnostic Systems Laboratories Inc.) with the following modifications to improve E2 delectability. An additional standard value was included ( $37 \mathrm{pmol} / \mathrm{l})$ to allow reliable detection below the lowest standard value provided by the kit $(74 \mathrm{pmol} / \mathrm{l})$. The protocol was also modified with respect to sample incubation $\left(20 \mathrm{~h}\right.$ at $7{ }^{\circ} \mathrm{C}$ instead of $3 \mathrm{~h}$ at $37^{\circ} \mathrm{C}$ ). The quantification was always conducted in parallel with positive controls with very low $(38 \mathrm{pmol} / \mathrm{l})$ and very high (3613 pmol/l) E2 values. Knockout $(n=10)$ 
and wild-type littermates $(n=9)$ were killed after assessment of the estrous cycle stage and blood was drawn from the vena cava. Only animals showing at least three consecutive 4-day estrous cycles were included in the analysis. Adaptation to the procedure of estrous cycle determination was taken as a precaution to minimize stress-related phenomena on the day of serum sample collection.

\section{RT-PCR}

Total RNA was isolated from adrenal glands using RNeasy Mini Kit (Qiagen). One microgram total RNA was then reverse transcribed using oligo-dT primer and the Superscript II Reverse Transcriptase kit (Promega). A total of 50 ng cDNA were subsequently amplified by PCR using 1.5U Taq polymerase (Invitrogen Life Technologies), 20 pmol of each primer (Akr1b8-fwd 5'-TgACCAAggCAgAATCCTCACC-3' and Akr1b8-rev 5'-AggATgTCAggCAggCTgggC-3'), $0.25 \mathrm{mM}$ dNTPs, $1.5 \mathrm{mM} \mathrm{MgCl}_{2}$ in a $50 \mu \mathrm{l}$ reaction volume at $94{ }^{\circ} \mathrm{C}$ for $3 \mathrm{~min}, 94{ }^{\circ} \mathrm{C}$ for $45 \mathrm{~s}, 62{ }^{\circ} \mathrm{C}$ for $45 \mathrm{~s}$, and $72{ }^{\circ} \mathrm{C}$ for $45 \mathrm{~s}$ with 28 cycles. Semi-quantitative conditions were established using increasing amounts of RNA. Products were separated on a $1.2 \%$ agarose gel. Expression levels of $A k r 1 b 8$ were compared with Hprt as a housekeeping control gene (primers sequences: Hprtfwd 5'-gCTggTgAAAAggACCTCT-3' and Hprt-rev $5^{\prime}$-CACAggACTAgAACACCTg- $3^{\prime} ; 57{ }^{\circ} \mathrm{C}$ annealing temperature, reaction conditions as mentioned earlier). Experiments were conducted in three replicates using extracted RNA pooled from adrenal glands of four animals respectively.

\section{Statistical analysis}

The significance of differences between groups was assessed using Student's $t$-test and $\chi^{2}$-test (for the evaluation of Mendelian ratios). Data are presented as the mean with variation between values indicated as S.E.M. Differences were considered significant when $P<0.05$.

\section{Results}

\section{Akr1b7 mRNA is strongly upregulated in theca and stroma cells during proestrus}

The induction of $A k r 1 b 7$ expression has been shown to occur within 1.5-6 h following hCG administration in immature mice and expression ceases $14 \mathrm{~h}$ post-hCG injection (Brockstedt et al. 2000). In sexually mature mice, LH is released within the proestrus phase briefly before ovulation occurs, with the highest serum concentrations occurring around $1800 \mathrm{~h}$ (Bailey 1987). To investigate whether the observed $\mathrm{LH}$-analog responsiveness occurs in vivo, $A k r 1 b 7 \mathrm{mRNA}$ expression in diestrus, proestrus, estrus, and metestrus ovaries was investigated by in situ hybridization. High levels of $A k r 1 b 7$ mRNA were detected in ovaries obtained from mice in proestrus $6 \mathrm{~h}$ postassumed LH surge (Fig. 1B), while no transcripts were detectable in ovaries during diestrus, estrus, metestrus, and proestrus at the approximate time of the $\mathrm{LH}$ surge or $14 \mathrm{~h}$ after (Fig. 1A, C and D). Akr1b7 mRNA expression in proestrus was restricted to theca and interstitial cells of the ovary. In antral follicles, only cells of the theca interna were stained; whereas in preantral follicles, $A k r 1 b 7$ transcripts were detected within the entire theca cell layer. In contrast, Akr1b7 mRNA was never detected in granulosa cells or in
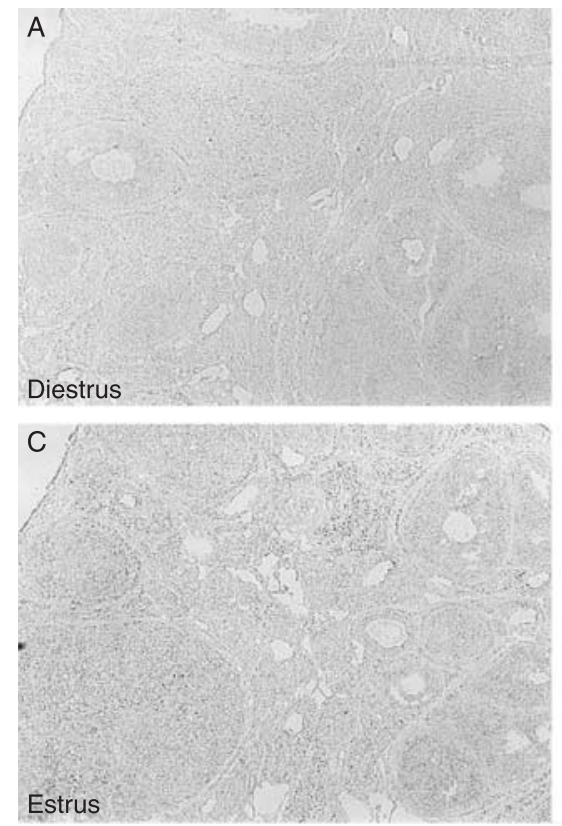

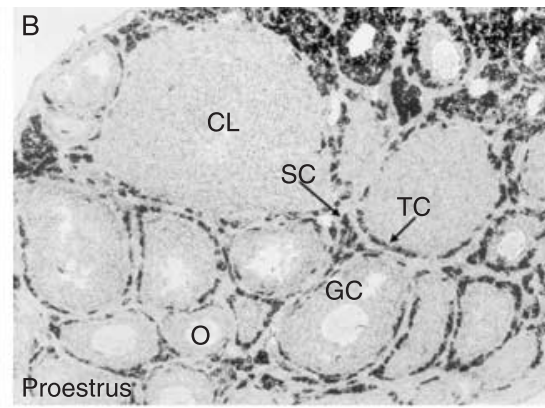

D

Metestrus
Figure 1 Non-radioactive in situ hybridization demonstrates ovarian Akr1b7 mRNA expression is restricted to the proestrus stage of the estrous cycle. $A k r 1 b 7$ expression was analyzed using in ovarian sections from mice in (A) diestrus, (B) proestrus (6 h post-endogenous LH surge), (C) estrus, and (D) metestrus. Akr1b7 mRNA is only expressed in theca and stromal cells of ovaries during proestrus. CL, corpus luteum; GC, granulosa cells; TC, theca cells; SC, stroma cells; $\mathrm{O}$, oocyte (Original magnification $\times 100$ ). 
corpora lutea (Fig. 1). Thus, the spatial distribution of Akr1b7 expression in the ovary correlates entirely with our previous data obtained following hCG administration (Brockstedt et al. 2000). However, the timeline of Akr1b7 expression under physiological conditions was more restricted to a period around $6 \mathrm{~h}$ post-assumed LH surge and had ceased within $14 \mathrm{~h}$. Furthermore, Akr1b7 mRNA was not detectable in progesterone-producing corpora lutea of pregnant mice by in situ hybridization. No evidence for $A k r 1 b 7$ mRNA transcripts, as assessed by northern blot analysis, could be found in the uterus of immature, eCG-treated mice before or after hCG administration or in the placenta from pregnant mice 13 days post-coitum (data not shown).

\section{Targeted disruption of the murine Akr1b7 gene}

The restricted expression of $A k r 1 b 7$ in proestrus ovary suggests that this gene product may play an important role in female reproductive physiology, particularly in the ovulatory process. Furthermore, the strong expression level reported in the vas deferens also suggests a role for $A k r 1 b 7$ in male reproduction. To investigate the effects on reproduction in vivo, knockout mice were prepared using homologous recombination in ES cells together with Cremediated loxP recombination.

Following the targeted insertion of a loxP site $5^{\prime}$ of exon 2 and a floxed neomycin selection cassette $3^{\prime}$ of exon 4, transient transfection of Cre recombinase in targeted ES cells led to the deletion of exons 2-4 (Fig. 2A), encoding the majority of the amino acids forming the substrate-binding domain (Jez et al. 1997, Hyndman et al. 2003). The disruption of the Akr1b7 gene at the genomic level was confirmed by Southern blot analysis (Fig. 2B). The effect of the gene deletion on $A k r 1 b 7$ transcripts was assessed using northern blot analysis of $A k r 1 b 7$-expressing organs. An $A k r 1 b 7$ cDNA probe detected a truncated transcript in knockout animals, suggesting that splicing from exons 1 to 5 occurs from the transgenic allele (Fig. 2C). No AKR1B7 protein could be detected in knockout mice, confirming the gene deletion (Fig. 2D). Genotyping of the litters of heterozygous intercrosses demonstrated the presence of wild-type, knockout, and heterozygous offspring at the expected Mendelian ratio (females: knockout $=50$, wild type $=51$, heterozygous $=96, \chi=0.39(\mathrm{df}=1)$, and
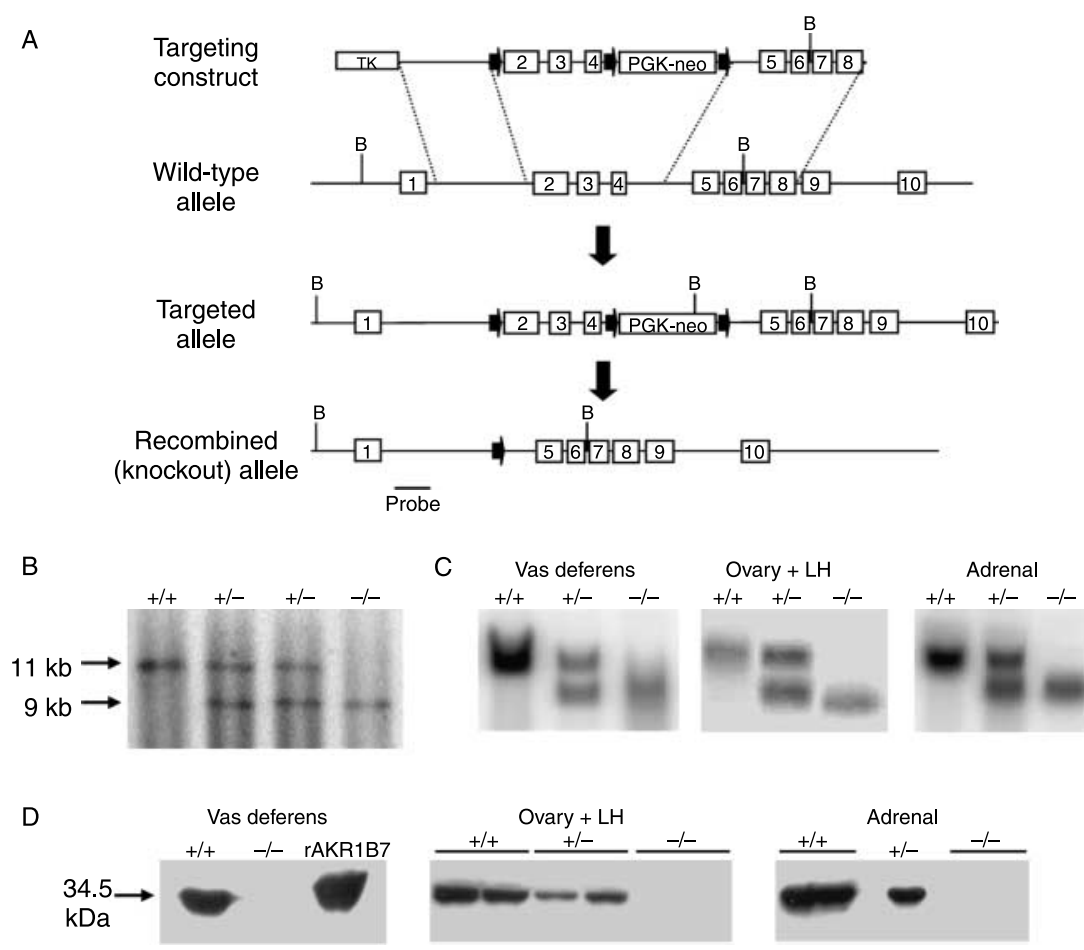

Figure 2 Targeted disruption of Akr1b7. (A) The targeting vector used to introduce a loxP site (indicated by black arrows) and a floxed selection cassette (PGK-neo) $5^{\prime}$ and $3^{\prime}$ of exons $2-4$ is shown together with the wild-type allele. A negative selection cassette (MC1-tk) used to enrich for homologous recombination was incorporated into the targeting vector. Following transient Cre transfection of homologously recombined ES cell clones, a loxP-mediated deletion occurs, removing exons 2-4 in the knockout allele. Exons appear as numbered boxes and the position of the probe and the BglII (B) restriction sites are indicated. (B) Southern analysis of BgllII-digested genomic DNA from wild-type $(+/+)$, heterozygote $(+/-)$, and knockout mice (-/-). (C) Northern blot analysis of $A k r 1 b 7$ expression in vas deferens, ovary ( $8 \mathrm{~h}$ post-hCG injection), and adrenal gland RNA from wild-type, heterozygote, and knockout mice demonstrates the presence of a truncated transcript in heterozygous and knockout animals. (D) Western analysis of AKR1B7 expression in vas deferens, $\mathrm{LH}$-stimulated ovary, and adrenal gland from wild-type, heterozygous, and knockout mice. Full length AKR1B7 protein is not detectable in knockout animals confirming deletion of targeting domain. Recombinant AKR1B7 (rAKR1B7) was used as a positive control. 
$P>0.05$; males: knockout $=53$, wild type $=49$, heterozygous $=106, \chi=0.89(\mathrm{df}=1)$, and $P>0.05)$, indicating that the mutation is not associated with embryonic lethality. Knockout mice appeared phenotypically normal when compared with their heterozygous and wild-type littermates, without obvious detrimental effects on health and viability. All phenotypic observations described were confirmed in $A k r 1 b 7$ knockout mice generated from three independent ES cell clones.

\section{Akr1b7 knockout animals reveal no histological abnormalities in reproductive organs and adrenal gland}

Histological analysis of reproductive organs revealed no abnormalities (Fig. 3A-D). Thus, despite the exceptionally high levels of AKR1B7 protein expression within the vas deferens and the strong $\mathrm{LH}$-dependent regulation in the ovary, apparently normal reproductive tissue histology was observed in the absence of functional AKR1B7 protein. Within the adrenal gland, no obvious morphological differences could be seen between the cortical zona fasciculata in knockout and wild-type littermates
(Fig. 3E and F). Furthermore, comparison of mean organ weights of the adrenal gland of male and female mice, the ovaries, the testes, and the epididymides revealed no apparent alteration in knockout animals when compared with wild-type littermates (Table 1).

\section{Reproductive function in Akr1b7 knockout mice is indistinguishable from wild-type littermates}

Despite the lack of apparent histological differences in mice lacking functional $A k r 1 b 7$, the effects of the mutation on reproductive success were investigated. Male and female wild-type and knockout mice were mated with wild-type animals continually throughout their reproductive life span and litter size together with the frequencies of parturitions were ascertained (Table 2). No significant differences $(P>0.05)$ were recorded between wild-type or knockout mice suggesting AKR1B7 protein to be dispensable for reproductive function.

Continuous mating of female mice from sexual maturity onwards results in repeated pregnancies with only few
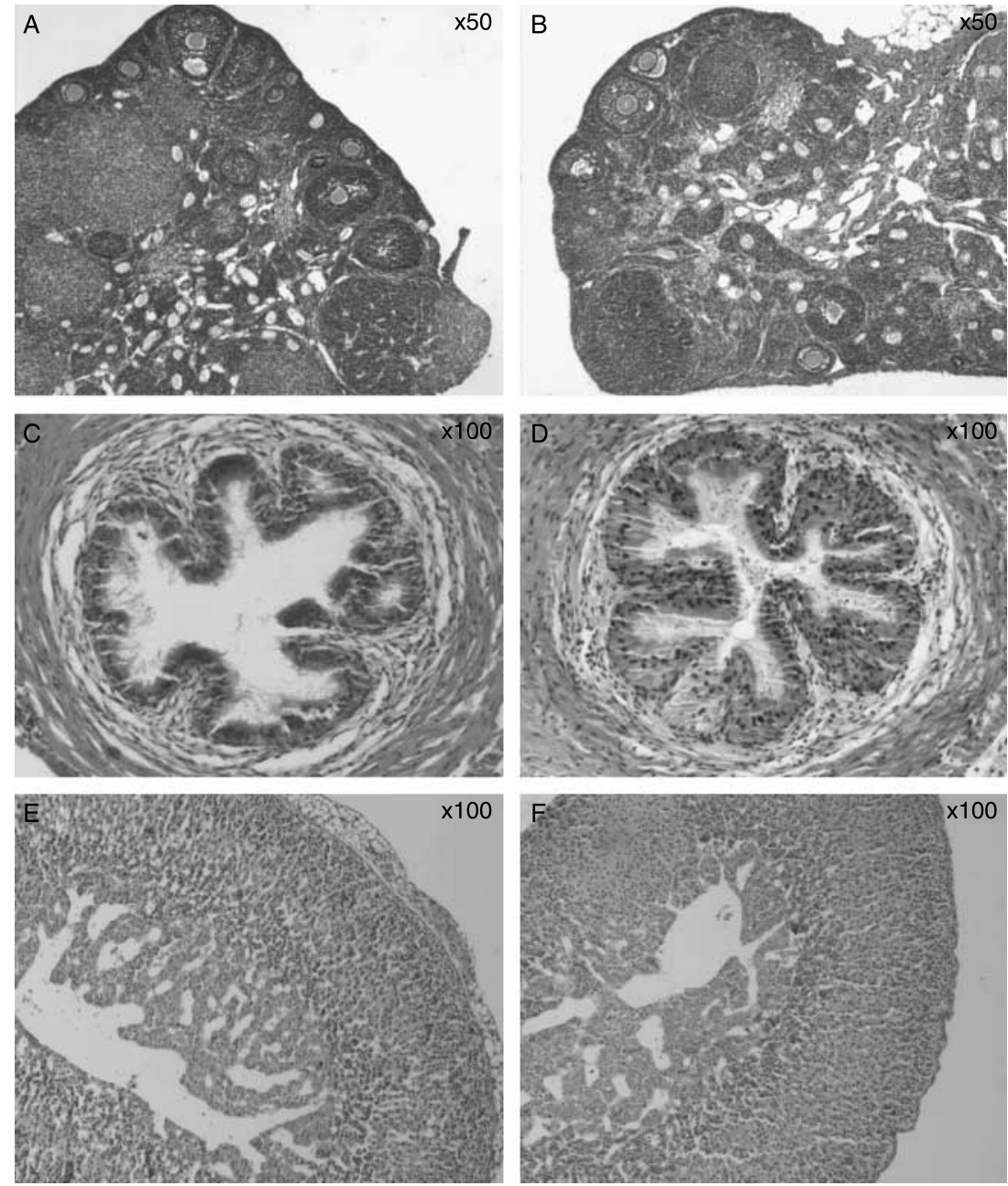

Figure 3 Histological analysis of AKR1B7expressing tissues. Photomicrographs of hematoxylin- and eosin-stained sections through the ovary (A, knockout; B, wild type), vas deferens (C, knockout; D, wild type), and adrenal gland ( $\mathrm{E}$, knockout; $\mathrm{F}$, wild type). Histology shown is from 20-week-old animals. 
Table 1 Organ weights are not significantly altered in $A k r 1 b 7$ knockout mice in comparison with wild-type littermate controls.

\begin{tabular}{|c|c|c|c|c|c|}
\hline & Genotype & Adrenal glands (mg) & Testes (mg) & Epididymides (mg) & Ovaries (mg) \\
\hline \multirow[t]{2}{*}{ Male } & Wild-type $(n=10)$ & $7.85 \pm 0.78$ & $125.7 \pm 4.0$ & $48.1 \pm 2.4$ & - \\
\hline & Knockout $(n=8)$ & $7.66 \pm 0.79 *$ & $126.3 \pm 8.2^{*}$ & $49.5 \pm 4.3^{*}$ & - \\
\hline \multirow{2}{*}{ Female } & Wild-type $(n=9)$ & $7.77 \pm 0.21$ & - & - & $12.22 \pm 1.12$ \\
\hline & Knockout $(n=13)$ & $7.96 \pm 0.36^{*}$ & - & - & $12.69 \pm 0.82 *$ \\
\hline
\end{tabular}

Data are presented as the mean \pm S.E.M. $* P>0.05$.

intermediate estrous cycles. Since $A k r 1 b 7$ expression in the ovary is restricted to a distinct period following the $\mathrm{LH}$ surge, the effects of the $A k r 1 b 7$ gene deletion may be simply masked by the reduced ovulation frequency in permanently mated mice. To test this hypothesis, knockout and wild-type littermates were mated only after they had reached 5 months of age. Housing females in the presence of male pheromones previous to mating thereby ensured maintenance of the estrous cycle. No effect $(P>0.05)$ of genotype on the reproductive performance of these mice was observed (Table 2).

\section{Estrous cycle and follicle maturation appears normal in Akr1b7 knockout mice despite distorted progesterone levels}

To further investigate the effects of $A k r 1 b 7$ mutation on ovarian function, serum concentrations of progesterone and estradiol were determined by RIA, and the duration of the estrous cycle and follicular maturation were assessed in knockout and wild-type littermates. No significant differences in hormone concentration of estradiol were observed in sera from wild-type and knockout mice during the estrus stage of the cycle (data not shown). However, progesterone levels were found to be significantly elevated $(P>0.05)$ in knockout animals $(32.5 \pm 4.7 \mathrm{nmol} / \mathrm{l} ; n=10)$ during metestrus when compared with wild-type littermates $(16.4 \mathrm{nmol} / \mathrm{l} \pm 3.2$; $n=9$ ) and significantly decreased during estrus in knockout animals $(12.9 \mathrm{nmol} / \mathrm{l} \pm 1.8 ; n=6)$ when compared with wild-type littermates $(18.9 \mathrm{nmol} / \mathrm{l} \pm 4.6 ; n=6)$. The estrous cycle stages of mice at 60 days of age were determined daily and monitored over a period of 15 days. No significant differences were found between knockout and wild-type littermates (Fig. 4A). Since ovarian follicles undergo a characteristic maturation process from primordial to antral stages of the leading follicles, the quantitative assessment of follicle maturation might provide a measure of subtle alterations in mice lacking functional AKR1B7 protein. Despite altered serum concentrations of progesterone in estrus (decreased) as well as metestrus (elevated) $A k r 1 b 7$ knockout mice exhibit no alterations of the ovarian follicle population in comparison with wild-type littermates, indicating that the mutation of $A k r 1 b 7$ does not adversely affect ovarian function (Fig. 4B) and female fertility. The lack of a detoxifying enzyme could impair cell survival and thus be associated with follicular atresia. However, although our experimental approach limited the assessment to early stages of follicular atresia, a strong atretic phenotype resulting from the targeted deletion would likely influence the overall distribution of non-atretic stages of follicular development; the distributions observed were indistinguishable between knockout and control litter mates, thus ruling out this possibility.

\section{Akr1b7 knockout males reveal normal spermatozoa parameters}

Since male mice are considered to be superfertile, the assessment of functional fertility parameters is a poor reflection of spermatozoa quality and quantity. Subsequently, the potential vas deferens effects of $A k r 1 b 7$ mutation on spermatozoa were assessed. Spermatozoa were isolated from the cauda region of the epididymis and the vas deferens of knockout and wild-type mice 22 weeks of age. No significant differences $(P>0.05)$ were found in sperm number or quality, as assessed by the proportion of motile spermatozoa as well as their absolute mobility between knockout and wild-type littermates. A slight regression in the percentage of motile sperm was recognized in samples from the vas deferens of knockout animals, which, although not being statistically significant $(P>0.05)$, demands further investigation (Table 3). Furthermore, a quantification of morphological abnormalities was conducted to

Table 2 Reproductive success of $A k r 1 b 7$ knockout mice at various ages is not impaired in comparison with wild-type littermate controls.

\begin{tabular}{|c|c|c|c|c|}
\hline & $\begin{array}{l}\text { Age at } \\
\text { time of } \\
\text { mating }\end{array}$ & Genotype & Litter size & Litters per month \\
\hline \multirow[t]{2}{*}{ Male } & \multirow[t]{2}{*}{6 weeks } & $\begin{array}{l}\text { Wild-type } \\
(n=13)\end{array}$ & $12.8 \pm 0.9$ & $0.82 \pm 0.13$ \\
\hline & & $\begin{array}{l}\text { Knockout } \\
(n=10)\end{array}$ & $12.3 \pm 1.4^{*}$ & $0.88 \pm 0.14^{*}$ \\
\hline \multirow[t]{4}{*}{ Female } & \multirow[t]{2}{*}{30 days } & $\begin{array}{l}\text { Wild-type } \\
(n=6)\end{array}$ & $7.11 \pm 0.94$ & $1.04 \pm 0.10$ \\
\hline & & $\begin{array}{l}\text { Knockout } \\
(n=10)\end{array}$ & $7.00 \pm 2.26^{*}$ & $1.02 \pm 0.23^{*}$ \\
\hline & \multirow[t]{2}{*}{5 months } & $\begin{array}{l}\text { Wild-type } \\
(n=5)\end{array}$ & $8.15 \pm 2.32$ & $1.07 \pm 0.25$ \\
\hline & & $\begin{array}{l}\text { Knockout } \\
(n=5)\end{array}$ & $8.12 \pm 1.73^{*}$ & $1.10 \pm 0.11^{*}$ \\
\hline
\end{tabular}

Data represent the mean litter size ( \pm S.E.M.) and the parturition frequency (litter per month ( \pm S.E.M. $)), * P>0.05$. 

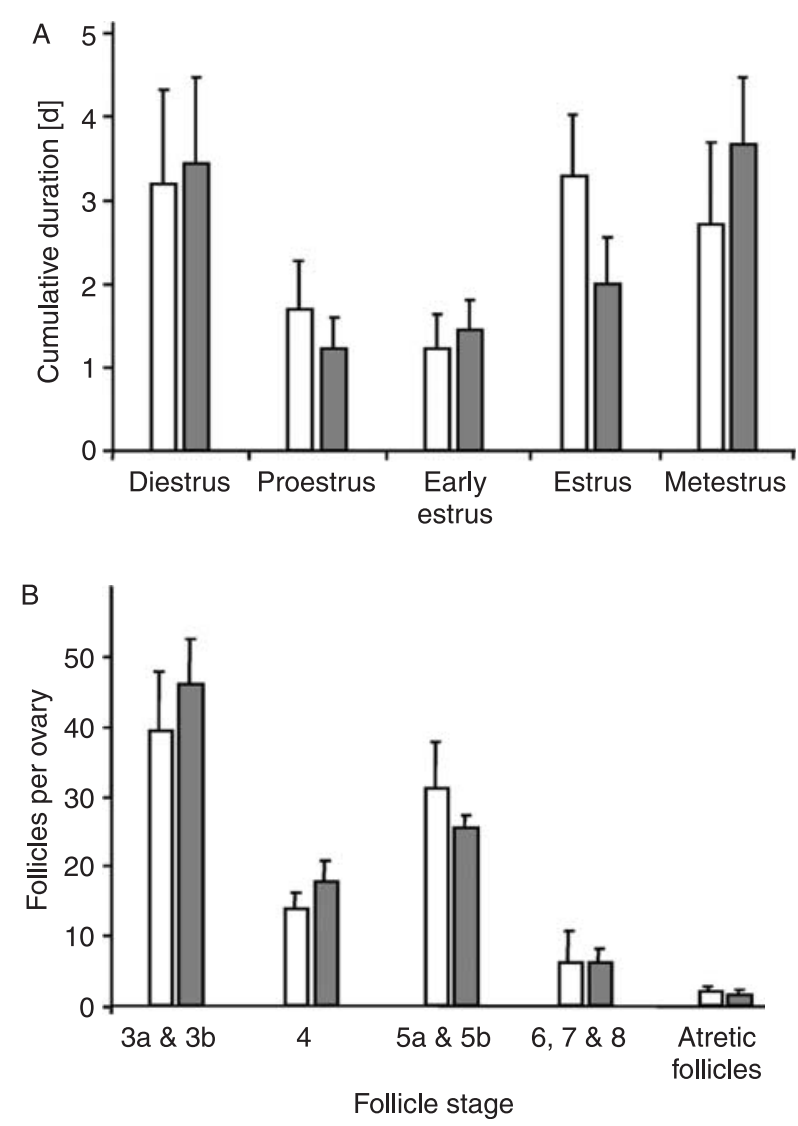

Figure 4 Estrous cycle duration and follicle maturation in AKR1B7 knockout mice are indistinguishable from wild-type littermates. (A) Estrous cycle stages were determined daily over a period of 15 days and are cumulatively summarized. Graphs represent the means \pm s.E.M. of knockout ( $n=9$; gray bars) and wild-type animals ( $n=9$; open bars). No significant differences between mutant and wild-type animals could be observed $(P>0.05)$. (B) Bars correspond to the number of follicles per ovary from knockout animals ( $n=4$; gray bars) when compared with wild-type littermates ( $n=4$; white bars). Classification was assessed according to Pedersen \& Peters (1968). Based on apoptotic characteristics, determination of atresia was restricted to stage 5 and antral follicles possessing intact nuclei. No significant differences between mutant and wild-type animals could be observed $(P>0.05)$.

determine the percentage of acephalic and head-to-head agglutinated spermatozoa (Table 4). For each parameter, no significant differences between wild-type and knockout animals were observed $(P>0.05)$. In single knockout animals, a slightly increased proportion of spermatozoa with abnormal morphological appearance was observed which, however, lacked statistical significance. Spermatozoa obtained from the non-AKR1B7 expressing cauda epididymis were used as an internal control, for comparisons of spermatozoa collected from the vas deferens and, as expected, no genotype-dependent differences were recorded.

\section{Compensatory effects: Akr1b8 expression levels are not elevated in Akr1b7 knockout animals}

AKR1B8 (fibroblast growth factor induced protein-1) is the structurally and functionally closest related protein to AKR1B7 (Seery et al. 1998). We therefore investigated whether $A k r 1 b 7$-expressing organs show elevated expression levels of $A k r 1 b 8$ mRNA by a compensatory mechanism. Northern blot analysis of ovarian and vas deferens $A k r 1 b 8$ transcripts and semi-quantitative RT-PCR of adrenal $A k r 1 b 8$ message revealed no apparent induction of $A k r 1 b 8$ expression in knockout animals when compared with wild-type littermates (Fig. 5). Specifically, Akr1b8 is reportedly not expressed in the vas deferens of wild-type mice. In $A k r 1 b 7$ knockout mice, no Akr1b8 mRNA was detected, contradicting a potential compensatory upregulation.

\section{Discussion}

Only little is known about the in vivo characteristics of AKR1B7 protein and its functional implication into reproductive processes. Within the present study, we first investigated the in vivo expression patterns of ovarian Akr1b7 mRNA during the natural 4-day estrous cycle of mice and demonstrated a rapid induction of $A k r 1 b 7 \mathrm{mRNA}$ following the proestrus stage of the cycle. Confirming our previous results using exogenous $\mathrm{LH}$-analog application, $A k r 1 b 7 \mathrm{mRNA}$ expression is sharply restricted to theca and interstitial cells following the preovulatory LH surge in vivo. However, while Akr1b7 expression following exogenous administration of an $\mathrm{LH}$ analog was found to be at a maximum after $14 \mathrm{~h}$, the results of this in vivo study revealed the highest expression levels $6 \mathrm{~h}$ after the approximate endogenous LH surge, whereas expression had ceased by $14 \mathrm{~h}$. We hypothesize that exogenous hCG administration with doses used in superovulation

Table 3 Spermatozoa parameters for Akr1b7 knockout mice and wild-type littermate controls ${ }^{a}$.

\begin{tabular}{|c|c|c|c|c|c|c|}
\hline & \multicolumn{3}{|c|}{ Cauda epididymis } & \multicolumn{3}{|c|}{ Vas deferens } \\
\hline & $\begin{array}{c}\text { Sperm count } \times \\
10^{6} / \text { cauda }\end{array}$ & Sperm motility (\%) & Mobility rank & $\begin{array}{l}\text { Sperm count } \times \\
10^{6} \text { /vas deferens }\end{array}$ & Sperm motility (\%) & Mobility rank \\
\hline Wild-type $(n=10)$ & $7.2 \pm 1.2$ & $67.8 \pm 7.5$ & $2.4 \pm 0.2$ & $4.3 \pm 1.4$ & $93.2 \pm 0.09$ & $2.9 \pm 0.03$ \\
\hline Knockout $(n=8)$ & $4.1 \pm 2.8^{*}$ & $60.3 \pm 12.5^{*}$ & $2.2 \pm 0.37^{*}$ & $7.0 \pm 1.2^{*}$ & $72.8 \pm 10.6^{*}$ & $2.8 \pm 0.15^{*}$ \\
\hline
\end{tabular}

Data are presented as the mean \pm s.E.M. $* P>0.05$.

${ }^{a}$ Data were obtained from animals 22 weeks of age. Motility is defined as the percentage of motile spermatozoa. Mobility (the extent or degree of movement) was assessed using an arbitrary scale $(0=$ no mobility to $3=$ normal mobility). 
Table 4 Spermatozoa morphology of $A k r 1 b 7$ knockout mice and wild-type littermate controls ${ }^{\mathrm{a}}$.

\begin{tabular}{|c|c|c|c|c|c|c|}
\hline & \multicolumn{3}{|c|}{ Cauda epididymis } & \multicolumn{3}{|c|}{ Vas deferens } \\
\hline & $\begin{array}{c}\text { Acephalic } \\
\text { spermatozoa (\%) }\end{array}$ & $\begin{array}{c}\text { Angulated } \\
\text { spermatozoa (\%) }\end{array}$ & $\begin{array}{c}\text { Head-to-head } \\
\text { agglutination (\%) }\end{array}$ & $\begin{array}{c}\text { Acephalic } \\
\text { spermatozoa (\%) }\end{array}$ & $\begin{array}{c}\text { Angulated } \\
\text { spermatozoa (\%) }\end{array}$ & $\begin{array}{c}\text { Head-to-head } \\
\text { agglutination (\%) }\end{array}$ \\
\hline Wild-type $(n=10)$ & $0.4 \pm 0.1$ & $0.4 \pm 0.4$ & $1.7 \pm 0.1$ & $0.6 \pm 0.3$ & $0.3 \pm 0.4$ & $1.7 \pm 0.9$ \\
\hline Knockout $(n=8)$ & $1.7 \pm 1.2^{*}$ & $2.8 \pm 4.5^{*}$ & $4.7 \pm 1.4^{*}$ & $1.5 \pm 1.1^{*}$ & $1.6 \pm 3.4^{*}$ & $5.5 \pm 1.9 *$ \\
\hline
\end{tabular}

Data are presented as the mean \pm S.E.M. ${ }^{*} P>0.05$.

${ }^{\mathrm{a} D a t a}$ were obtained from animals 22 weeks of age.

experiments results in higher serum hormone levels that are maintained for longer periods of time in the circulatory system and result in prolonged mRNA expression.

This distinct expression profile supports the assumption that the AKR1B7 enzyme might play a critical role in the ovulation process, possibly by neutralizing the buildup of toxic intermediates and byproducts of $\mathrm{LH}$-induced steroidogenesis. Following the LH surge, isocaproaldehyde, a preferred substrate of AKR1B7, is produced through a gonadotropin-enhanced P450scc (CYP11A1) activity. Therefore, AKR1B7 might fulfill an isocaproaldehyde detoxifying function not only in the adrenal cortex but also in ovarian theca and stroma cells.

To investigate the potential role of AKR1B7 protein in ovulatory processes, we generated mutant $A k r 1 b 7$ mice. Due to the abundant adrenal expression of AKR1B7 which potentially fulfils a vital role in detoxification processes within this organ, a deletion strategy was chosen which would allow maximal flexibility should a lethal phenotype be observed. Using a Cre/loxPapproach two types of recombinant clones were obtained for both, the generation of a constitutive knockout lacking AKR1B7 in all tissue types and of a conditional knockout for restricted genetic manipulation of a particular tissue.

Contrary to expectations, constitutive deletion of Akr1b7 resulted in viable mice, which appeared phenotypically normal in comparison with their wild-type littermates. Furthermore, a lack of statistical variation from the expected Mendelian ratios resulting from heterozygous intercrosses, confirmed that the $A k r 1 b 7$ mutation is compatible with normal growth and development, despite the reported embryonic expression of this gene in the actively steroidogenic embryonic adrenal gland (Lau et al. 1995). This finding is in contrast to the results of Lefrancois-Martinez who demonstrated that stable transfection of adrenal Y1 cells with $A k r 1 b 7$ antisense cDNA led to decreased viability when the cells were exposed to the AKR1B7 substrate isocaproaldehyde and also when increased steroidogenesis was induced within the cells (Lefrancois-Martinez et al. 1999). This conflict demonstrates the importance of an in vivo approach for functional gene analysis. Since the adult and embryonic adrenal gland is a well-known site of expression of a diverse array of AKR family members (Maser \& Bannenberg 1994, Lau et al. 1995), the potential detoxifying role of AKR1B7 may be replaced in the mutant mouse by a related enzyme. Potentially, these compensatory mechanisms are not preserved in the aforementioned in vitro models. Despite the lack of a demonstrable reproductive phenotype in this work, future studies will investigate the effects of $A k r 1 b 7$ deletion following physiological challenge, conditions which are potentially associated with an increased requirement for detoxifying AKR1B7 enzyme.

As shown by the determination of diverse parameters of female reproductive function, such as litter size, parturition frequency, estrous progression, and follicle staging analysis, AKR1B7 expression is dispensable for ovarian function. Furthermore, recurrent ovulations without intermitting pregnancies, and thus, the longterm effects of the gene disruption did not have an impact on female fertility and no alterations in female reproductive lifespan were observed. Alterations found in serum hormone concentrations of progesterone in metestrus- and estrus-stage knockout females seemingly failed to affect reproductive performance. Progesterone is mainly synthesized following rupture of the Graafian follicle in the corpus luteum. However, other tissues producing progesterone include the placenta, theca cells of early follicle stages, and the adrenal gland. Since AKR1B7 protein expression is restricted to theca cells shortly before ovulation (Brockstedt et al. 2000) and to the zona fasciculata of the adrenal gland (Lau et al. 1995,

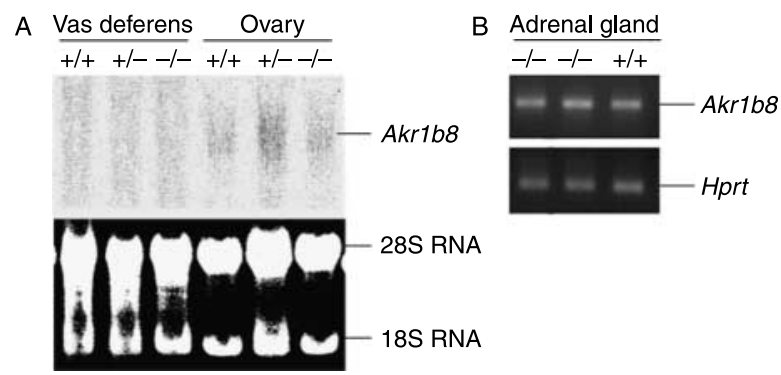

Figure 5 Akr1b8 mRNA expression analysis of $A k r 1 b 7$ knockout animals. Ovarian and vas deferens tissue was examined by northern blot analysis (A). No induction of mRNA expression in knockout animals when compared with wild-type littermates was observed. Importantly, no Akr1b8 mRNA was detected in the vas deferens of knockout males. The $28 \mathrm{~S}$ and $18 \mathrm{~S}$ rRNAs were used as loading controls. Adrenal $A k r 1 b 8$ expression was investigated by semi-quantitative RT-PCR with no apparent differences in Akr1b8 expression between knockout and wild-type animals (B). Expression of Hprt was used as a loading control. 
Aigueperse et al. 1999), effects of the targeted deletion of $A k r 1 b 7$ in the adrenal gland could possibly interfere with hormonal feedback mechanisms affecting hormone levels depending on the estrous cycle stage of $A k r 1 b 7$ knockout animals. Furthermore, malfunction of the adrenal gland could have effects on metabolic processes rather than reproductive performance. These hypotheses deserve further investigation.

A gross assessment of spermatozoa collected from the vas deferens of male knockout mice similarly confirmed that male reproductive function is also independent of AKR1B7 protein expression within this organ. However, a trend towards decreased motility in knockout mice was observed and might indicate subtle differences missed due to the arbitrary scale analysis used in the present study. This deserves a more detailed investigation. However, a degree of variation in fertility parameters (including sperm motility) has been reported between different mouse strains (Silver 1995) and the observed motility difference may simply be due to the mixed genetic background used in this study, resulting from the use of 129 strain ES cells. The establishment of this mutation on different purebred genetic backgrounds would help the quantification of a potential reproductive phenotype. Indeed, the use of a sub-fertile strain such as 129 could perhaps reveal a fertility phenotype as yet masked by the $\mathrm{C} 57 \mathrm{BL} / 6$ strain used in this study.

The lack of an obvious impairment of spermatozoa is surprising while considering the high proportion of AKR1B7 protein within the soluble protein fraction of vas deferens. It has, however, been shown that this high level of AKR1B7 expression is unique for the vas deferens of the mouse when compared with other rodent species (Taragnat et al. 1990, Schnolzer et al. 1992, Aigueperse et al. 1999). The murine expression has been shown to be the result of a $77 \mathrm{bp}$ LINE element-derived sequence insertion, which is specific to the murine AKR1B7 promoter and conveys androgen responsiveness. This observation has led to a discussion concerning the selective advantage of a high AKR1B7 expression level in the murine vas deferens (Val et al. 2004). One explanation suggested an involvement of AKR1B7 in prostaglandin synthesis, which has been shown to be comparatively high within the murine vas deferens (Badr 1975, Marshburn et al. 1989). The results of this study, however, suggest that functional AKR1B7 protein is dispensable for normal reproductive function of the murine vas deferens, indicating that any selective advantage is subtle.

The observed lack of a reproductive phenotype in both male and female knockout mice, along with the presence of other AKRs within reproductive tissues, suggested that compensatory mechanisms might be at play. We therefore investigated the possible induction of Akr1b8 expression - the structurally and functionally closest related protein (Seery et al. 1998). Significantly, we found no indication for an expression of $A k r 1 b 8$ in the vas deferens of neither knockout nor wild-type animals. While, in contrast to $A k r 1 b 7, A k r 1 b 8$ expression in the ovary is independent from $\mathrm{LH}$, in the adrenal gland and in the post-LH ovary $A k r 1 b 7$ and $A k r 1 b 8$ are expressed at similarly high levels. The lack of an observed strong upregulation of $A k r 1 b 8$ in these tissues argues against the presence of a compensatory mechanism involving this related family member.

Since fertility serves the purpose of species maintenance, the selective pressure for such mechanisms within reproductive physiology is high. Therefore, several distinct defense systems presumably act together and might compensate for a defective mechanism. Other members of the AKR gene family, besides Akr1b8, as well as enzymes with similar properties, e.g., glutathione-S-transferases, are likely candidates which could replace some of the functions of AKR1B7.

In addition to the potential role in detoxification of isocaproaldehyde resulting from steroidogenesis, a role for this AKR in oxidative stress is suggested by its substrate specificity for 4-HNE. This toxic intermediary is released following lipid peroxidation and is considered to be a central mediator of the toxic effects resulting from oxidative stress. Besides the cytotoxic characteristics of 4-HNE endangering cell viability and causing pathological alterations, for example, in disorders such as atherosclerosis (Palinski et al. 1989, Rosenfeld \& Ross 1990) and neurodegenerative diseases (Yoritaka et al. 1996, Ando et al. 1998, Calingasan et al. 1999), this compound has also been shown to induce a variety of cellular response processes, e.g., apoptosis, cell growth, detoxification, and protein turnover (Uchida 2003 and references therein). Furthermore, when compared with free radicals, 4-HNE is relatively stable and through diffusion can act upon target cells far away from the original source of the lipid peroxidation (Dianzani et al. 1999). Therefore, a potential physiological role for AKR1B7 in the control of 4-HNE levels may have effects in a far more subtle manner than expected, leading to diverse effects on cellular physiology. Recently, an association of AKR1B7 protein with the intestinal nuclear receptor liver $X$ receptor (LXR), acting as sterol sensor (Repa \& Mangelsdorf 2002, Volle et al. 2004), has been reported. These interactions and the multiple functions of adrenal glucocorticoids in inflammatory, energy, and stress reactions are starting points for future experiments which may reveal a potential role of AKR1B7 protein in non-reproductive tissues.

\section{Acknowledgements}

This work was supported by Bayer Schering Pharma AG, Berlin, Germany. The authors wish to thank Dagmar Sonnenberg for her excellent technical assistance. The authors declare that there is no conflict of interest that would prejudice the impartiality of this scientific work. 


\section{References}

Aigueperse C, Martinez A, Lefrancois-Martinez A, Veyssiere G \& Jean C 1999 Cyclic AMP regulates expression of the gene coding for a mouse vas deferens protein related to the aldo-keto reductase superfamily in human and murine adrenocortical cells. Journal of Endocrinology 160 147-154.

Aitken R 1994 A free radical theory of male infertility. Reproduction, Fertility, and Development 6 19-23.

Ando $Y$, Brännström T, Uchida K, Nyhlin N, Näsman B, Suhr O, Yamashita T, Olsson T, Salhy ME, Uchino M et al. 1998 Histochemical detection of 4-hydroxynonenal protein in Alzheimer amyloid. Journal of the Neurological Sciences 156 172-176.

Badr F 1975 Prostaglandin levels in tissues of the male reproductive system in six strains of mice. Endocrinology 96 540-543.

Bailey K 1987 Diurnal progesterone rhythms in the female mouse. Journal of Endocrinology 112 15-21.

Bohren K, Bullock B, Wermuth B \& Gabbay K 1989 The aldo-keto reductase superfamily. cDNAs and deduced amino acid sequences of human aldehyde and aldose reductases. Journal of Biological Chemistry 264 9547-9551.

Brockstedt E, Peters-Kottig M, Badock V, Hegele-Hartung C \& LessI M 2000 Luteinizing hormone induces mouse vas deferens protein expression in the murine ovary. Endocrinology 141 2574-2581.

Burstein S \& Gut M 1971 Biosynthesis of pregnenolone. Recent Progress in Hormone Research 27 303-349.

Butcher RL, Collins WE \& Fugo NW 1974 Plasma concentration of LH, $\mathrm{FSH}$, prolactin, progesterone and estradiol-17 $\beta$ throughout the 4-day estrous cycle of the rat. Endocrinology 94 1704-1708.

Calingasan NY, Uchida K \& Gibson GE 1999 A novel marker of oxidative stress in Alzheimer's disease. Journal of Neurochemistry 72 751-756.

Comporti M 1998 Lipid peroxidation and biogenic aldehydes: from the identification of 4-hydroxynonenal to further achievements in biopathology. Free Radical Research 6 623-635.

Constantopoulos G, Carpenter A, Satoh P \& Tchen T 1966 Formation of isocaproaldehyde in the enzymatic cleavage of cholesterol side chain by adrenal extract. Biochemistry 5 1650-1652.

Davies B, Baumann C, Kirchhoff C, Ivell R, Nubbemeyer R, Habenicht UF, Theuring F \& Gottwald U 2004 Targeted deletion of the epididymal receptor HE6 results in fluid dysregulation and male infertility. Molecular and Cellular Biology 24 8642-8648.

Dianzani M, Barrera G \& Parola M 1999 4-Hydroxy-2,3-nonenal as a signal for cell function and differentiation. Acta Biochimica Polonica 46 61-75.

Esterbauer H, Schaur R \& Zollner H 1991 Chemistry and biochemistry of 4-hydroxynonenal, malonaldehyde and related aldehydes. Free Radical Biology and Medicine 11 81-128.

Goldring N, Durica JM, Lifka J, Hedin L, Ratoosh SL, Miller WL, Orly J \& Richards JS 1987 Cholesterol side-chain cleavage P450 messenger ribonucleic acid: evidence for hormonal regulation in rat ovarian follicles and constitutive expression in corpora lutea. Endocrinology 120 1942-1950.

Hegele-Hartung C, Siebel P, Peters O, Kosemund D, Muller G, Hillisch A, Walter A, Kraetzschmar J \& Fritzemeier KH 2004 Impact of isotype-selective estrogen receptor agonists on ovarian function. PNAS 101 5129-5134.

Hyndman D, Bauman D, Heredia V \& Penning TM 2003 The aldo-keto reductase superfamily homepage. Chemico-Biological Interactions 143-144 621-631.

Jez J, Flynn T \& Penning T 1997 A new nomenclature for the aldo-keto reductase superfamily. Biochemical Pharmacology 54 639-647.

Laemmli U 1970 Cleavage of structural proteins during the assembly of the head of bacteriophage T4. Nature 227 680-685.

Lau ET, Cao D, Lin CS, Chung S \& Chung S 1995 Tissue-specific expression of two aldose reductase-like genes in mice: abundant expression of mouse vas deferens protein and fibroblast growth factor-regulated protein in the adrenal gland. Biochemical Journal 312 609-615.
Lefrancois-Martinez A-M, Tournaire C, Martinez A, Berger $M$, Daoudal S, Tritsch D, Veyssiere G \& Jean C 1999 Product of sidechain cleavage of cholesterol, isocaproaldehyde, is an endogenous specific substrate of mouse vas deferens protein, an aldose reductase-like protein in adrenocortical cells. Journal of Biological Chemistry 274 32875-32880.

Marshburn P, Clark M \& Shabanowitz R 1989 Immunohistochemical localization of prostaglandin $\mathrm{H}$ synthase in the epididymis and vas deferens of the mouse. Biology of Reproduction 41 491-497.

Martinez A, Aigueperse C, Val P, Dussault M, Tournaire C, Berger M, Veyssiere G, Jean C \& Lefrancois Martinez A 2001 Physiological functions and hormonal regulation of mouse vas deferens protein (AKR1B7) in steroidogenic tissues. Chemico-Biological Interactions 130-132 903-917.

Maser E \& Bannenberg G 1994 11-beta-Hydroxysteroid dehydrogenase mediates reductive metabolism of xenobiotic carbonyl compounds. Biochemical Pharmacology 47 1805-1812.

Palinski W, Rosenfeld ME, Yla-Herttuala S, Gurtner GC, Socher SS, Butler SW, Parthasarathy S, Carew TE, Steinberg D \& Witztum JL 1989 Low density lipoprotein undergoes oxidative modification in vivo. PNAS 86 1372-1376.

Pedersen T \& Peters H 1968 Proposal for a classification of oocytes and follicles in the mouse ovary. Journal of Reproduction and Fertility 17 555-557.

Pizzimenti S, Laurora S, Briatore F, Ferretti C, Dianzani M \& Barrera G 2002 Synergistic effect of 4-hydroxynonenal and PPAR ligands in controlling human leukemic cell growth and differentiation. Free Radical Biology and Medicine 32 233-245.

Repa JJ \& Mangelsdorf DJ 2002 The liver X receptor gene team: potential new players in atherosclerosis. Nature Medicine 8 1243-1248.

Richards J, Hedin L, Lifka J, Ratoosh S, Durica J \& Goldring NB 1987 Ovarian follicular development: from physiology to molecular biology. Recent Progress in Hormone Research 43 231-276.

Rosenfeld M \& Ross R 1990 Macrophage and smooth muscle cell proliferation in atherosclerotic lesions of $\mathrm{WHHL}$ and comparably hypercholesterolemic fat-fed rabbits. Arteriosclerosis 10 680-687.

Rugh R 1968 In The Mouse, Its Reproduction and Development, pp 3839. Minneapolis: Burgess Publishing Company.

Schnolzer M, Alewood P, Jones A, Alewood D \& Kent S 1992 In situ neutralization in boc-chemistry solid phase peptide synthesis. rapid, high yield assembly of difficult sequences. International Journal of Peptide and Protein Research 40 180-193.

Seery L, Nestor P \& FitzGerald G 1998 Molecular evolution of the aldo-keto reductase gene superfamily. Journal of Molecular Evolution 46 139-146.

Silver L 1995 Mouse Genetics. Concepts and Applications, New York: Oxford University Press.

Smith A 1991 Culture and differentiation of embryonic stem cells. Journal of Tissue Culture Methods 13 89-94.

Taniguchi H \& Hayashi N 1998 A liquid chromatography/electrospray mass spectrometric study on the post-transcriptional modification of tRNA. Nucleic Acids Research 26 1481-1486.

Taragnat C, Berger M \& Jean C 1986 Identification and androgendependence of proteins in the mouse vas deferens. International Journal of Andrology 9 299-311.

Taragnat C, Berger M \& Jean C 1988 Preliminary characterization, androgen-dependence and ontogeny of an abundant protein from mouse vas deferens. Journal of Reproduction and Fertility 83 835-842.

Taragnat C, Berger M \& Jean C 1990 Tissue and species specificity of mouse ductus deferens protein. Journal of Andrology 11 279-286.

Uchida K 2003 4-Hydroxy-2-nonenal: a product and mediator of oxidative stress. Progress in Lipid Research 42 318-343.

Val P, Aigueperse C, Ragazzon B, Veyssiere G, Lefrancois-Martinez A-M \& Martinez A 2004 Adrenocorticotropin/3', 5'-cyclic AMP-mediated 
transcription of the scavenger $a k r 1-b 7$ Gene in adrenocortical cells is dependent on three functionally distinct steroidogenic factor-1responsive elements. Endocrinology 145 508-518.

Volle DH, Repa JJ, Mazur A, Cummins CL, Val P, Henry-Berger J, Caira F, Veyssiere G, Mangelsdorf DJ \& Lobaccaro J-MA 2004 Regulation of the aldo-keto reductase gene $a k r 1 b 7$ by the nuclear oxysterol receptor LXR $\alpha$ (liver X receptor- $\alpha$ ) in the mouse intestine: putative role of LXRs in lipid detoxification processes. Molecular Endocrinology 18 888-898.

World Health Organization 1987 World Health Organization Laboratory Manual for the Examination of Human Semen and Semen-Cervical Mucus Interaction, Cambridge, UK: Cambridge University Press.

Yang X, Li C, Xu X \& Deng C 1998 The tumor suppressor SMAD4/DPC4 is essential for epiblast proliferation and mesoderm induction in mice. PNAS 95 3667-3672.
Yi P, Zhan D, Samokyszyn V, Doerge D \& Fu P 1997 Synthesis and 32Ppostlabeling/high-performance liquid chromatography separation of diastereomeric 1,N2-(1,3-propano)-2'-deoxyguanosine 3'-phosphate adducts formed from 4-hydroxy-2-nonenal. Chemical Research in Toxicology 11 1259-1265.

Yoritaka A, Hattori N, Uchida K, Tanaka M, Stadtman E \& Mizuno Y 1996 Immunohistochemical detection of 4-hydroxynonenal protein adducts in Parkinson disease. PNAS 93 2696-2701.

Received 13 January 2007

First decision 6 February 2007

Accepted 10 April 2007 